\title{
Computational Evaluation of Airframe Noise Reduction Concepts at Full Scale
}

\author{
Mehdi R. Khorrami* \\ NASA Langley Research Center, Hampton, Virginia, 23681 \\ and \\ Benjamin Duda ${ }^{\dagger}$, Andreas Hazir ${ }^{\ddagger}$, and Ehab Fares ${ }^{\S}$ \\ Exa GmbH, Curiestrasse 4, D-70563 Stuttgart, Germany
}

\begin{abstract}
High-fidelity simulations focused on full-scale evaluation of new technologies for mitigating flap and landing gear noise are presented. These noise reduction concepts were selected because of their superior acoustic performance, as demonstrated during NASA wind tunnel tests of an $18 \%$-scale, semi-span model of a Gulfstream aircraft. The full-scale, full-aircraft, time-accurate simulations were performed with the lattice Boltzmann PowerFLOW $^{\circledR}$ solver for free air at a Mach number of 0.2. Three aircraft configurations (flaps deflected at $3^{\circ}$ without and with main gear deployed, and $0^{\circ}$ flaps with main gear extended) were used to determine the aeroacoustic performance of the concepts on component-level (individually) and system-level (concurrent application) bases. Farfield noise spectra were obtained using a Ffowcs-Williams and Hawkings acoustic analogy approach. Comparison of the predicted spectra without (baseline) and with the noise treatments applied showed that noise reduction benefits between 2-3 dB for the flap and 1.3-1.7 dB for the main landing gear are obtained. It was also found that the full extent of the benefits is being masked by the noise generated from the flap brackets and main gear cavities, which act as prominent secondary sources.
\end{abstract}

\section{Introduction}

Alleviation of the adverse environmental impact aircraft noise causes on population centers near airports is a high priority goal of the NASA Aeronautics Research Mission Directorate. As air travel is projected to grow steadily within the next two decades, ${ }^{* *}$ novel concepts that will make next generation civil transports exceptionally quiet must be introduced. Airframe noise is a prominent component of aircraft noise during landing. Wing high-lift devices, such as slats and flaps, and the aircraft undercarriage system are major sources of airframe noise. ${ }^{1-6}$ Presently, system-level development and evaluation of viable airframe noise reduction technologies in a relevant environment can only be achieved with full-scale flight tests. The NASA Environmentally Responsible Aviation (ERA) project aimed to advance and accelerate the development of simulation-based, system-level, airframe noise prediction tools that can be applied at full scales. Maturation of such tools will help promote a leap in aircraft design from the current timeconsuming and costly "cut-and-try" approach to a physics-based, virtual design environment where aeroacoustic evaluation/optimization of a noise reduction concept can take place in an integrated fashion.

Computational simulations have made significant inroads as a viable, complementary tool to wind tunnel testing for airframe noise prediction. These complex, high-fidelity simulations were mostly confined to sub- or full-scale airframe components. ${ }^{7-11}$ As part of the NASA-Gulfstream partnership on airframe noise, time-accurate aeroacoustic simulations were performed on an $18 \%$-scale, semi-span, high-fidelity model of a Gulfstream aircraft without and with flap and landing gear noise mitigation concepts installed. ${ }^{12-15}$ These model-scale simulations represented a first attempt at the prediction of 1) system-level airframe noise for an aircraft in landing configuration and 2) aeroacoustic performance of numerous airframe noise mitigation concepts. Very good agreement between simulated results and wind tunnel measurements of steady and unsteady surface pressure fields, off-surface velocity field, and farfield acoustic behavior of the $18 \%$-scale aircraft model was demonstrated, establishing the validity of the computational approach outlined in references $13-16$ as a powerful predictive tool.

\footnotetext{
* Aerospace Engineer, Computational AeroSciences Branch, Associate Fellow AIAA

$\uparrow$ Senior Applications Engineer, Aerospace, Member AIAA

* Senior Applications Engineer, Aerospace Aeroacoustics, Member AIAA

$\S$ Senior Technical Director, Aerospace Applications, Senior Member AIAA

** https://www.faa.gov/data research/aviation/aerospace forecasts, cited April 8, 2016.
} 
The present study is part of a comprehensive computational effort designed to advance the state-of-the-art in airframe noise prediction from sub-scale models to full-scale, fully integrated configurations. Following our earlier model scale simulations, ${ }^{13-14}$ the current full-scale, full-aircraft airframe noise prediction study was executed via application of a lattice Boltzmann-Very Large Eddy Simulation (LB-VLES) approach within the Exa Corporation Pow$\mathrm{erFLOW}^{\circledR}$ solver. In this paper, we present aeroacoustic simulations of three aircraft configurations with noise reduction (NR) technologies applied to the flaps and main landing gear. To assess the performance of the NR technologies and their noise abatement benefits, the simulations were designed to provide an accurate resolution of the local flow fields at several locations: the flap tips, flap brackets, the regions near the main landing gear including the wheel cavity, and the gear-flap interaction zone. A requisite for successful aeroacoustic evaluation of the NR concepts is the availability of simulated results for untreated (baseline) configurations. The computational methodology described here was used earlier to predict the flow around baseline versions of the three cases used in the present effort. The resulting aeroacoustic data were validated using existing model- and full-scale measurements of the same aircraft. The baseline study is summarized in a companion paper by Khorrami and Fares. ${ }^{17}$

\section{Full-Scale Aircraft Geometry}

The simulated geometry corresponds to the Gulfstream aircraft used during the NASA-Gulfstream joint airframe noise flight test conducted in 2006. ${ }^{18}$ An $18 \%$-scale, semi-span, high-fidelity reproduction of the same aircraft was used for an extensive study of flap and landing gear noise sources and corresponding noise mitigation concepts. ${ }^{13-14}$ The geometry used for the present work was developed using a full-scale version of the $18 \%$-scale model fuselage, wing, and flow-through nacelle; the flaps, including their complex bracket/track system, as well as the vertical and horizontal tails, were developed using as-flown, full-scale geometry files furnished by the Gulfstream Aerospace Corporation (GAC). The main landing gear, including the wheel cavity, was developed from the original full-scale geometry files containing the finer details of the gear as deployed on the actual aircraft. The larger structures residing within the wheel well that could potentially alter the cavity volume were also included. Since the gear cavity extends across the fuselage, the full aircraft span had to be considered in the simulations. Thus, the computational cost and resources required for each aircraft configuration to be simulated were significantly higher than those used for the $18 \%$-scale, semi-span model simulations ${ }^{13,14}$ mainly because of the higher Re and inclusion of the full-span geometry.

For the purpose of evaluating the aeroacoustic performance of a select number of flap and gear noise abatement technologies, three aircraft configurations were identified as baselines. These were among the four configurations flown during the 2006 flight test (see Fig. 1) and served as baselines for evaluating the aeroacoustic performance of numerous flap and gear noise abatement concepts during the $18 \%$-scale model test. The configuration with the flaps deflected at $39^{\circ}$ and main gear retracted (Fig. 1a) was used to isolate the noise sources associated with the flap and to determine the simulated, system-level, aeroacoustic performance of a select number of flap tip treatments. ${ }^{14,19}$ The second configuration with the flaps at $0^{\circ}$ (cruise wing) and main gear deployed (Fig. 1b), although not a landing condition, was used to isolate the noise sources associated with the main landing gear and to determine the effectiveness of several gear fairings in reducing the noise from this component. ${ }^{14}$ The configuration with $39^{\circ}$ flap deflection and main landing gear deployed (Fig. 1c) represents a more complete aircraft geometry during landing and was used to determine the efficacy of the flap and gear noise mitigation technologies when applied concurrently. ${ }^{14,19}$
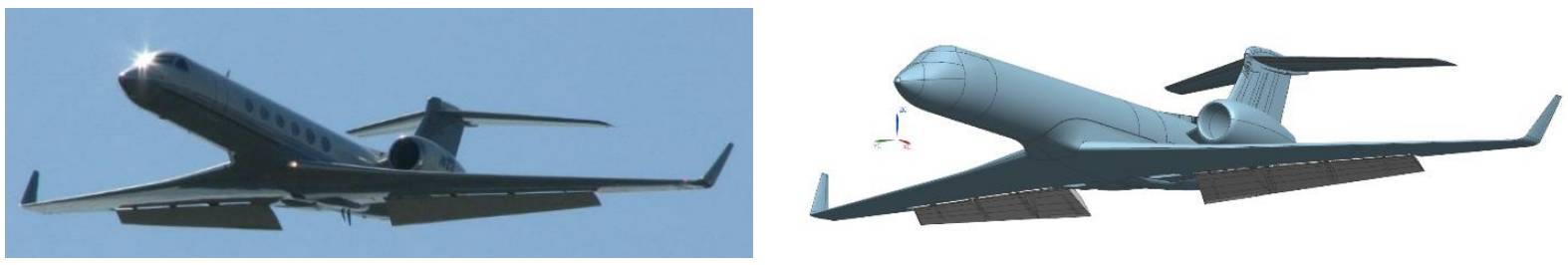

a) $39^{\circ}$ deflected flaps with main gear retracted. Left image, 2006 flight test and right image, simulated model.

Figure 1. Baseline configurations for full-scale Gulfstream aircraft. 

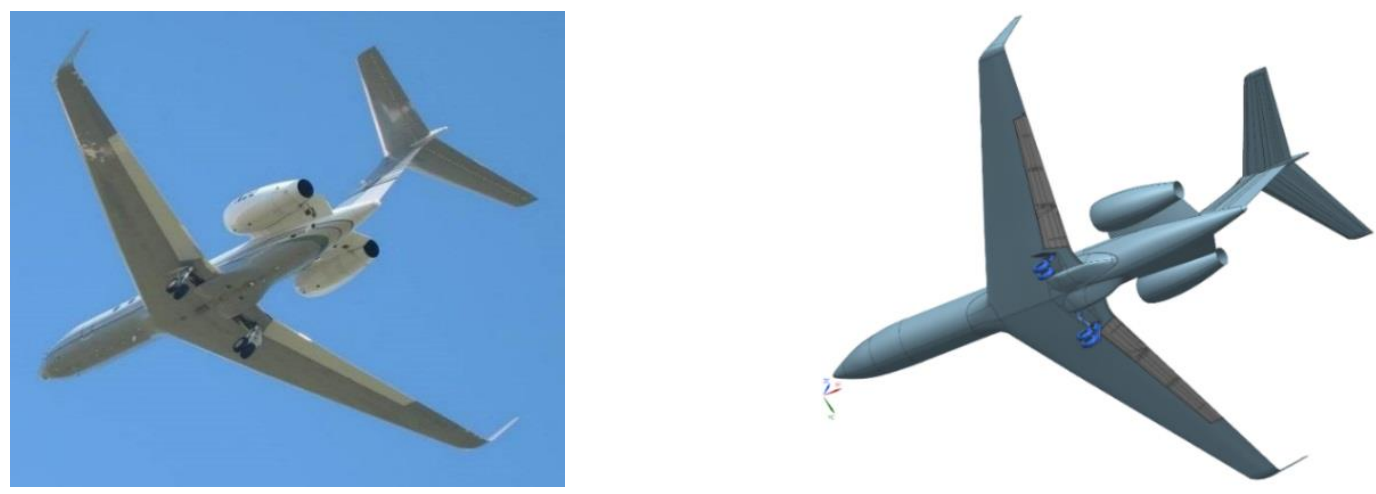

b) $0^{\circ}$ flaps (cruise wing) with main gear deployed. Left image, 2006 flight test and right image, simulated model.
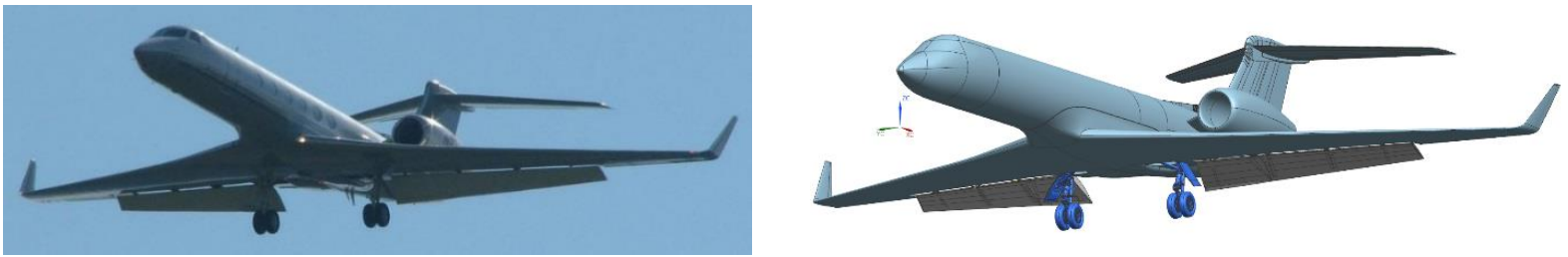

c) $39^{\circ}$ deflected flaps with main gear deployed. Left image, 2006 flight test and right image, simulated model.

\section{Figure 1. Concluded.}

The selected NR technologies were a subset of the concepts subjected to extensive evaluation during the 18\%scale model test. ${ }^{19}$ The technologies evaluated here are the FLEXible Side-Edge Link (FLEXSEL) and a porous surface treatment for the flap tips. FLEXSEL is a structural-link design that uses stretchable materials for linking the flap side edge to the adjacent wing edge. The porous surface treatment consists of replacing a small portion of the upper and lower surfaces, plus the side-edge surface at the flap inboard and outboard tips, with a porous surface having the desired flow resistance. For the main landing gear, the NR technologies comprise a porous knee fairing (PKF) and an assortment of smaller fairings that are collectively referred to as "upper fairings" (UF). These represent some of the best performing devices, and except for the FLEXSEL, all were among the concepts downselected for implementation on the Gulfstream test aircraft of a now-cancelled flight test campaign. For our computational evaluation, we used the full scale designs of the porous surface treatment, PKF, and UF concepts that passed the Gulfstream-NASA critical design review process. The porous flap side edges consist of several metallic sheets with laser-drilled perforations. The CAD model of the inboard and outboard flap side edges, as well as the porous sheets, are presented in Fig. 2. The inboard and outboard patches are characterized by a resistance of 570 Rayls and 280 Rayls, respectively. Figure 3 depicts the full-scale main landing gear with the PKF and UF concepts installed. The full-scale FLEXSEL shown in Fig. 4 was created using the $18 \%$-scale version of the device that was tested on the semi-span aircraft model.
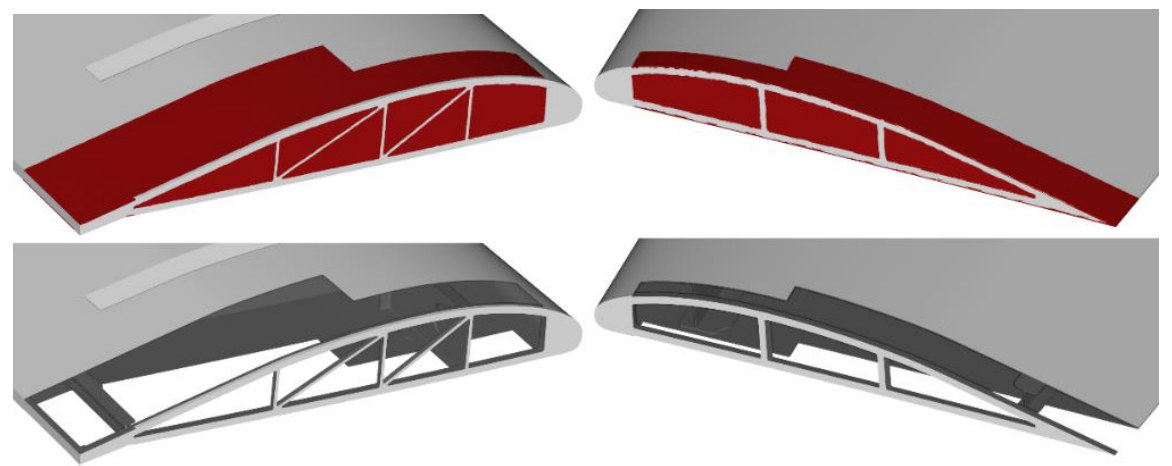

Figure 2. Porous flap side edges: inboard (left), outboard (right); flap with porous patches (top), flap alone (bottom). 

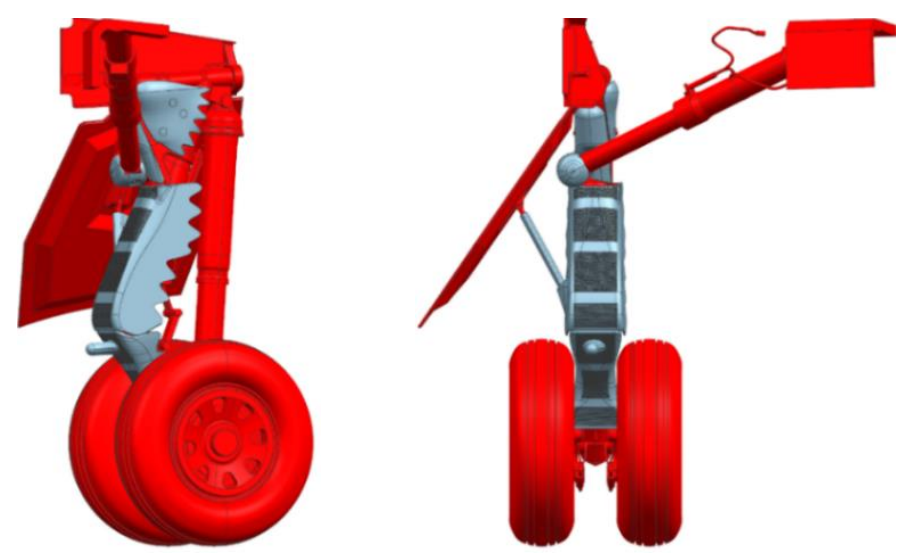

Figure 3. Full-scale versions of upper and porous knee fairings.

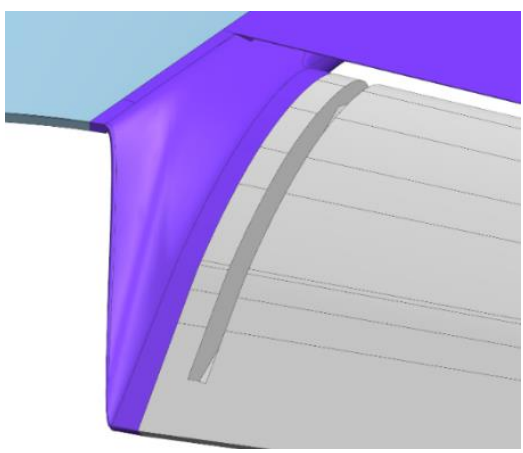

a) Inboard

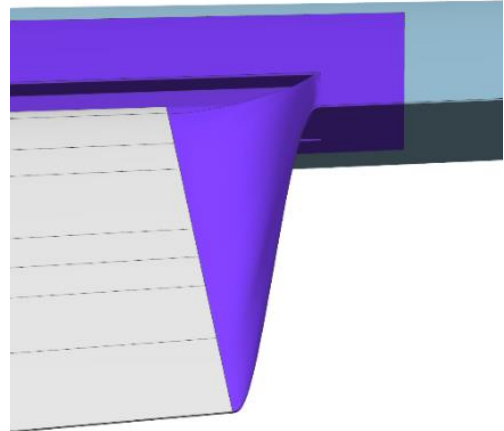

b) Outboard

Figure 4. Full-scale FLEXSEL based on $18 \%$-scale model.

\section{Computational Approach}

The computational approach used in the present effort is a direct extension of the methodology outlined in references 13 and 14 to an entire, full-scale aircraft. The numerical simulations were performed using the commercial flow solver PowerFLOW ${ }^{\circledR}$, which is based on the three dimensional 19 state (D3Q19) lattice Boltzmann model (LBM). The software has been successfully validated for flows around highly complex airframe geometries such as those reported in references 7-9, 11, and 13-15.

\section{A. Flow Solver}

The lattice Boltzmann equations represent an alternative mesoscopic formulation to the classical macroscopic Navier-Stokes equations describing a compressible unsteady flow of a continuum. The details of the mathematical foundations are documented in references 9, 11 and 20. The simple, local formulation of the underlying D3Q19 equations allows a highly efficient implementation for distributed computations on thousands of processors. The low dissipation and dispersion properties of the numerical scheme typically produce aerodynamic and aeroacoustic results that are comparable to those obtained with classical CFD solvers that use higher-order large eddy simulation (LES), as demonstrated in the comparative study of flow over tandem cylinders presented in reference 21 .

\section{B. Turbulence Modeling}

The lattice Boltzmann flow simulation is equivalent to a Direct Numerical Simulation (DNS) of the flow. For high Reynolds number flows, such as those addressed in this work, the lattice Boltzmann Very Large Eddy Simulation (LB-VLES) approach described in references 22 and 23 is used in conjunction with an extended wall function. The approach has been applied previously in references 9, 13, and 14 .

\section{Computational Grids}

The lattice Boltzmann formulation is solved on Cartesian meshes that are generated automatically within PowerFLOW $^{\circledR}$ for any geometrically complex shape. This greatly simplifies the labor-intensive volume meshing step usually 
associated with other approaches. The gridding strategy, overall mesh distribution, and arrangement of the variable resolution (VR) regions for various grids were described in references 13, 14, and 16. A study on the effects of spatial resolution was also conducted for most of the full-scale configurations reported in this paper. For consistency, however, only results on medium and fine (where noted) meshes will be presented.

\section{Boundary Conditions}

The simulations were performed for free air. All aircraft surfaces, except for the segments treated as porous, were modeled with no slip boundary conditions via a generalized bounce back volumetric formulation ${ }^{24,25}$ near the wall for arbitrarily oriented surfels within the voxels. For the regions where the surfaces were treated as porous, the Acoustic Porous Media (APM) boundary condition ${ }^{26,27}$ within the PowerFLOW ${ }^{\circledR}$ solver was invoked. Prior to usage in the full scale simulations, the APM boundary condition was vastly improved, rigorously tested via dedicated simulations of the $18 \%$-scale semi-span model, and validated extensively against available aeroacoustic measurements of the model with the porous surface treatment applied (unpublished results). Farfield boundary conditions for velocity, turbulent kinetic energy and pressure were used at the inflow and outflow boundaries. In addition, sponge layer zones were included in the far field to damp reflections of acoustic waves.

\section{Porous Surface Treatment for Flap Side Edges}

In the LB method, external forces can be included in the flow dynamics by altering the local, instantaneous particle distributions during the collision step. PowerFLOW ${ }^{\circledR}$ implements a porous medium model by applying an external force driven by the flow resistivity as a function of the local flow velocity. ${ }^{28}$ This model can be used to predict pressure losses that affect the time-averaged flow field solution and, at the same time, the pressure fluctuations caused by the impinging acoustic waves. The porous surface patches at the flap side edges are modeled by the APM boundary condition, a patented technology by Exa Corporation. ${ }^{29,30,31}$ The APM consists of an adiabatic porous medium covered by a face sheet that induces a pressure jump based on the given porosity and tortuosity. The physical properties of the APM condition (thickness, resistance, porosity and tortuosity) can be tuned to achieve a desired surface impedance and resistance. The later parameter was adjusted to obtain the resistances specified at the flap inboard and outboard tips, 570 Rayls and 280 Rayls, respectively.

\section{E. Computational Procedure}

The PowerFLOW ${ }^{\circledR}$ simulations were initialized with freestream conditions for a period corresponding to the time that it would take the flow to convect a distance of 40 mean aerodynamic chords. The time steps $(\Delta \mathrm{t})$ used for the current simulations were $5.44 \times 10^{-7} \mathrm{~s}, 8.15 \times 10^{-7} \mathrm{~s}$, and $1.22 \times 10^{-6} \mathrm{~s}$ for the fine, medium, and coarse grids, respectively. Statistical convergence of the flow solution during this initial transient time was monitored using unsteady pressure signals acquired on the surface of the wing and the time history of the global aerodynamic forces acting on the aircraft.

After reaching statistical convergence within the first $2.0 \mathrm{~s}$ of simulated physical time, the simulation was executed for an additional $1.0 \mathrm{~s}$ during which the computed flow variables were stored in a number of different volume and surface measurement files for subsequent flow analysis and post processing. The flow variables were recorded with various sampling frequencies (for the fine simulation) ranging from about $57 \mathrm{kHz}$ for the surface to $15 \mathrm{kHz}$ in some areas of the volume. Post-processing spectral analyses were conducted using a bandwidth of $3.6 \mathrm{~Hz}$ and an overlapping coefficient of 0.5 .

An acoustic analogy approach based on the Ffowcs-Williams and Hawkings (FWH) formulation ${ }^{32}$ was used to extrapolate the computed near-field fluctuations to the far field. The employed FWH formulation is based on the retarded-time formulation $1 \mathrm{~A}$ by Farassat ${ }^{33}$ extended to account for uniform mean flow convection effects to simulate the noise generated and measured in an ideal infinite wind tunnel. ${ }^{34}$

\section{Results and Discussion}

The computations presented here were executed at $M=0.2$. A Reynolds number $(\mathrm{Re})$ of $10.5 \times 10^{6}$, roughly half the flight Re (based on the full scale wing mean aerodynamic chord and aircraft landing speed), was chosen for the simulations. This value was selected after a modest study targeting Re effects revealed that beyond $\mathrm{Re}=10 \times 10^{6}$ scale effects on steady and unsteady surface pressure fields diminished significantly. ${ }^{16}$ Accordingly, we believe that the farfield noise levels obtained at this Re are nearly equivalent (at a lower computational cost) to the corresponding levels at the full flight Re. The original, full-scale flap brackets were found to be prominent secondary noise sources, contributing significantly to the farfield noise signature at medium and high frequencies and masking the true acoustic performance of the noise reduction concepts. A targeted study by Fares et al. ${ }^{16}$ revealed that the presence of various relatively small pin holes and openings in the brackets were the cause of the extra noise. Most of these small 
holes/openings were the result of missing screws, bolts, and other surface definition mismatches in the original fullscale geometry files/descriptions. As was shown in references 16 and 17, closing the openings removes most of the high-frequency resonances that are produced by the holes. Results obtained with the slightly modified brackets are presented in this paper.

\section{A. Global View}

A global picture of the simulated vorticity field for the landing configuration of $39^{\circ}$ and main gear retracted without (baseline) and with the FLEXSEL concept applied is presented in Fig. 5. Major regions of flow unsteadiness are clearly identified in Fig. 5a. As expected, the flap inboard and outboard tips produce high-amplitude flow fluctuations. Also prominent in this figure is the level of flow unsteadiness that is generated by the flap brackets. The corresponding field with the FLEXSEL concept installed is shown in Fig. 5b. Observe that the concept drastically alters the flow field at the tips, producing compact vortices that form and reside away from the flap surfaces. We must note that the flap brackets produce similar levels of unsteadiness with/without the FLEXSEL concept. However, in Fig. 5b the unsteadiness associated with the brackets appears weaker because of the higher value of vorticity assigned to the isosurfaces to better visualize the flow field near the flap tip regions. The vorticity field for the porous tip (APM) treatment is shown in Fig. 5c. Although difficult to discern from the figure, this concept substantially reduces the surface pressure fluctuations in the vicinity of the side edges. The effect that these NR technologies have on the unsteady pressure field at the flap inboard edge is clearly illustrated in Fig. 6. In this figure, the view is from the aircraft fuselage looking toward the wing tip. Observe that both FLEXSEL and the porous surface treatment significantly diminish the fluctuating pressures at the tip, relative to the baseline configuration. In the FLEXSEL case, a narrow strip of high amplitude pressure fluctuations at the trailing edge of the linkage still remains.

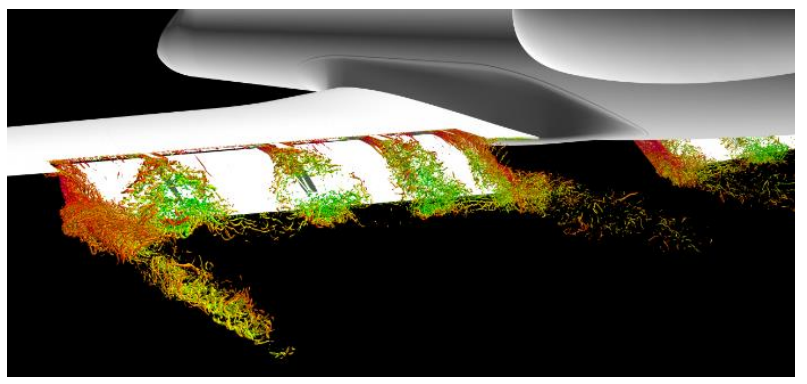

a) Baseline

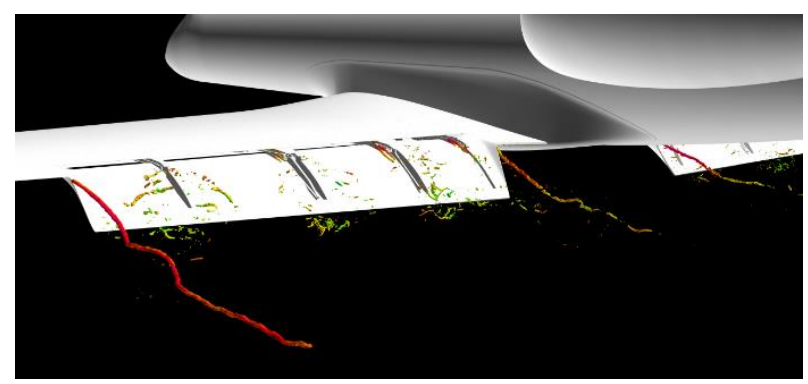

b) FLEXSEL

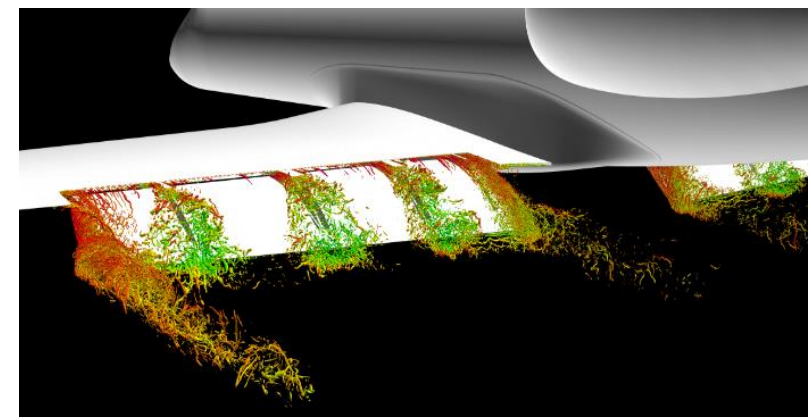

c) Porous surface (APM)

Figure 5. Instantaneous vorticity field based on isosurface of $\lambda_{2}$ criterion for configuration with $39^{\circ}$ flap deflection and main gear retracted. 


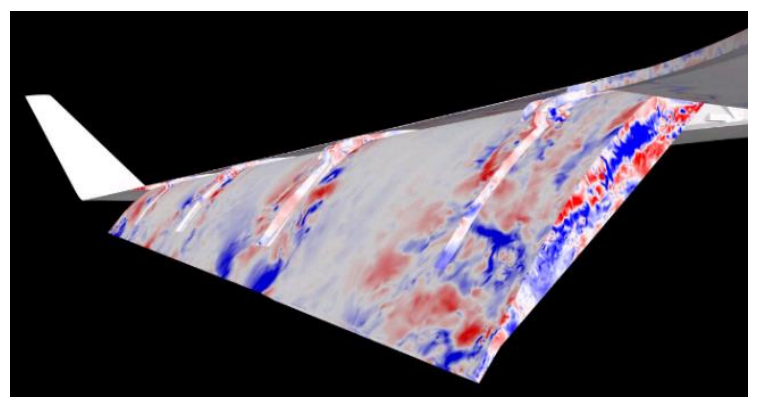

a) Baseline

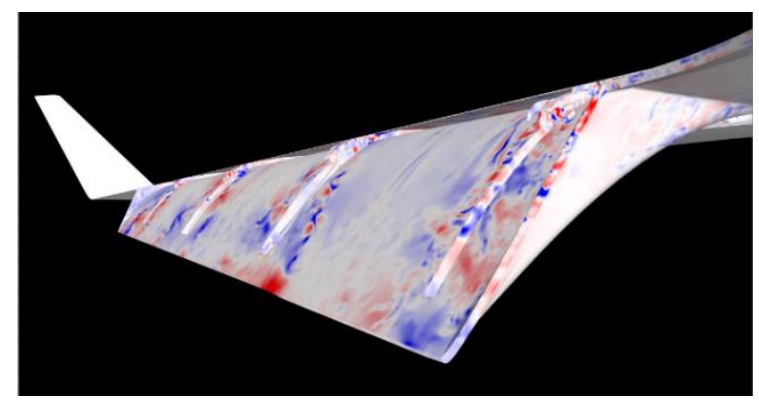

b) FLEXSEL

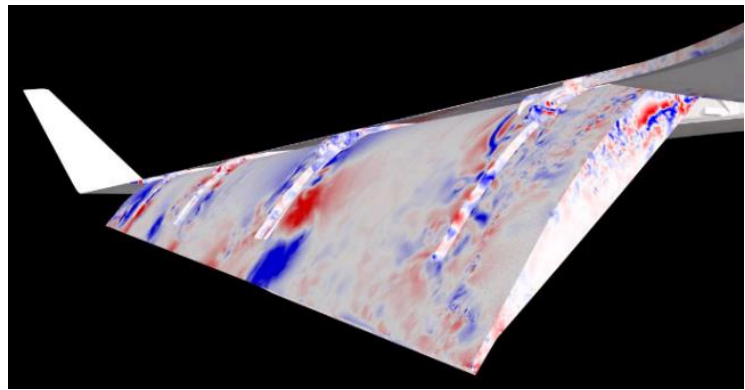

c) Porous surface (APM)

Figure 6. Instantaneous pressure field at inboard edge for configuration with $39^{\circ}$ flap deflection and main gear retracted.

A global view of the radiated sound field produced by this aircraft configuration is given in Fig. 7. The twodimensional planar cut, positioned near the flap leading edge, clearly identifies both inboard and outboard flap tips as the strongest sources of sound (Fig. 7a). Although weaker, the waves generated by the brackets are clearly discernible. As discussed by Fares et al., ${ }^{16}$ the flap brackets for this aircraft are important secondary sources contributing a measurable amount of noise in the mid- to high-frequency range. More detailed discussions on the relative importance of the noise associated with the flap brackets are provided in references 16 and 17. Also note from Fig. 7a the presence of asymmetry in the radiating pressure waves, indicating that a prominent tonal source exists in the vicinity of the flap outboard edge on the port (left) side of the aircraft. This asymmetry is physical and caused by the presence of two relatively small cavities on the port wing only, adjacent to the outboard flap side edge (see Fig. 8). In the actual aircraft, these cavities may be fully or partially open to a large interior reservoir. For simulation purposes, the cavity depths were limited to 5.725 inches based on the scant engineering drawings available. Thus, the characteristics of the tone (excitation, frequency, and amplitude) may not represent properly the phenomenon occurring on the actual aircraft. The acoustic field for FLEXSEL (Fig. 7b) shows a substantial reduction of flap tip noise by this technology. The dominant tone on the port wing has disappeared due to elimination of the cavities by the FLEXSEL device. The radiating pressure field with the porous tip concept applied is shown in Fig. 7c. This concept also reduces tip noise significantly. However, application of this treatment has no major effect on the cavity tone emanating from the port wing; thus, the pressure contours on that side of the aircraft indicate, erroneously, that no reduction in noise has been achieved. 


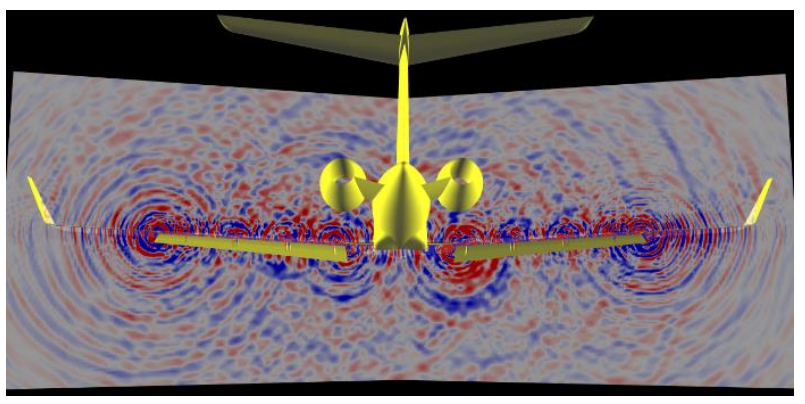

a) Baseline

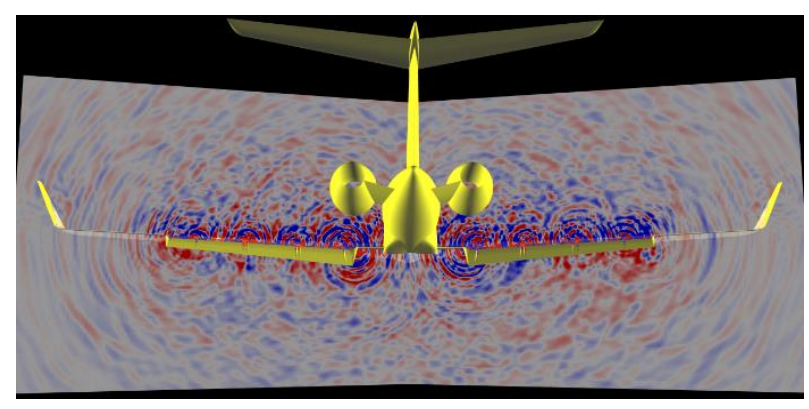

b) FLEXSEL

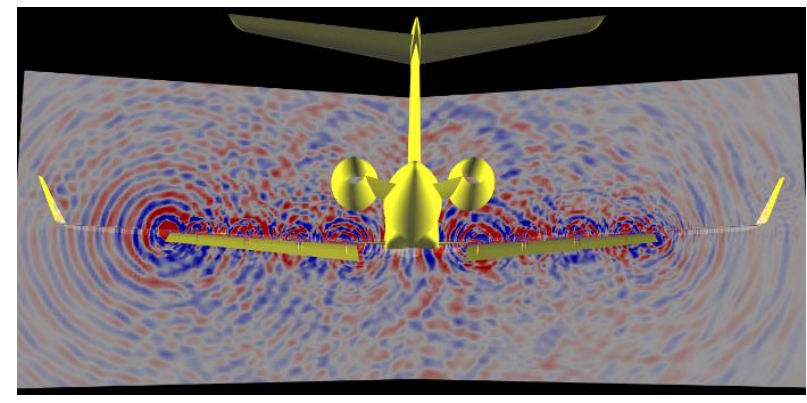

c) Porous surface (APM)

Figure 7. Radiated sound (dilation) field for configuration with $39^{\circ}$ flap deflection and main gear retracted (planar cut near flap leading edge).

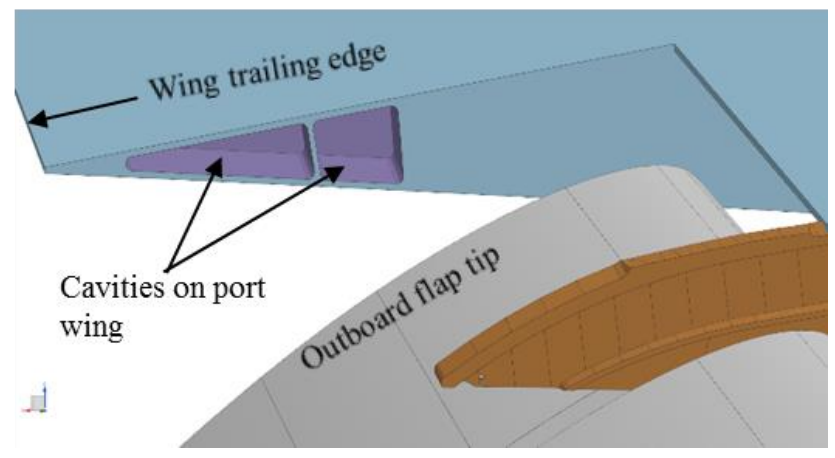

Figure 8. Tone-generating cavities on aircraft port wing.

\section{B. Flap Noise Reduction Concepts}

Time-averaged (static) surface pressure fields at the flap inboard and outboard tips are presented in Figs. 9 and 10, respectively. The contour plots serve to illustrate how each tip treatment alters formation of the flap side-edge vortices and thus their pressure imprint on the surface. As can be inferred, application of the porous surface treatment creates a significant drop in the suction imposed by the side edge vortices. In the case of the FLEXSEL concept, while the pressure imprints caused by the edge vortices have nearly disappeared due to a diminished roll-up process, a narrow strip of high suction remains at the trailing edge of the device where the flap links with the wing.

Based on integrated forces, the FLEXSEL concept was found to produce $~ 1 \%$ more lift and $2.1 \%$ less drag than the corresponding values for the baseline configuration. These levels were expected due to reduction in the induced drag at the flap tips. The differences in global lift and drag between the baseline and porous flap tips were found to be negligible. The computed forces for both flap concepts are very close to those measured during the $18 \%$-scale model test of the same aircraft. ${ }^{19,35}$ Spanwise lift distributions produced by the tip modifications for the complete aircraft and flap alone are shown in Fig. 11 for the starboard side. For the aircraft (Fig. 11a), slight differences are observed among the three simulated configurations. The lift distribution along the flap span (Fig. 11b) exhibits noticeable local differences between the treated and untreated flaps. As reflected in Fig. 11, at the inboard edge, the peak suction 
pressure caused by the tip vortex is substantially reduced by the FLEXSEL concept and nearly eliminated by application of the porous surface (APM) treatment. Notice that the latter concept also alters the flow around the brackets by reducing flow separation, thus producing a total lift value that is close to the baseline level (despite the loss of vortex lift). Instantaneous total pressure fields on a planar cut at the inboard tip near the flap trailing edge are displayed in Fig. 12. The FLEXSEL configuration produces a tightly-wound tip vortex that resides far above the solid surfaces and thus has a diminished pressure footprint. In contrast, the porous surface treatment produces a fairly large, diffused vortex with a weak core.

The farfield noise computation was conducted using an FWH propagation formulation ${ }^{28}$ and pressures on the solid surfaces of the complete, full-scale aircraft. The data sampling frequency for the pressure field on the solid surfaces was $50 \mathrm{kHz}$. Fig. 13 shows farfield sound pressure level (SPL) directivity maps calculated on the symmetry plane of the aircraft at a distance of $29.6 \mathrm{~m}$, for frequencies between $20 \mathrm{~Hz}$ and $8,000 \mathrm{~Hz}$. As can be observed, both concepts are quite effective in reducing the broad component of flap noise over the entire range of directivity angles. The predicted power spectral density (PSD) levels plotted in Fig. 14 were calculated for a farfield microphone located 394 feet $(120 \mathrm{~m})$ from the aircraft center of rotation (flyover certification point). Two major tones at frequencies of 170 $\mathrm{Hz}$ and $540 \mathrm{~Hz}$ are visible. The first tone is caused by resonance of the flow-through nacelle, which happens only for simulations involving the modified flap brackets. The reasons why closure of various small openings and holes on the flap brackets results in nacelle resonance are not yet clear to us. The second tone $(540 \mathrm{~Hz})$ is associated with the two cavities on the port wing discussed in the previous section. Since our main interest is the broadband component of airframe noise, all subsequent noise reduction values reported in this paper correspond to broadband noise, that is, tonal contributions to the spectral levels have been removed.

Returning to Fig. 14, observe that noise levels over the entire frequency range were lowered with application of the FLEXSEL concept. For a realistic assessment of NR concept acoustic performance, we have used a D-weighting scale (which accentuates high-frequency content and approximates PNL computations) to calculate farfield OASPL (overall sound pressure level). Based on D-weighted results, the reduction in OASPL produced by the FLEXSEL concept is approximately $2.5 \mathrm{dBD}$. In reality, however, the noise reduction effectiveness of this device is even greater. This cannot be appreciated in the figure because the true performance of the concept is being masked by the stillsubstantial level of high-frequency noise produced by the modified brackets, which is enhanced by D-weighting the spectra. The porous tip treatment is also efficient at lowering noise over the entire frequency range. Based on Dweighted results, the overall reduction achieved by this device was also close to $2.5 \mathrm{dBD}$. Fine resolution simulation of the porous tip treatment yielded even higher noise reduction levels, approaching $3.0 \mathrm{dBD}$.

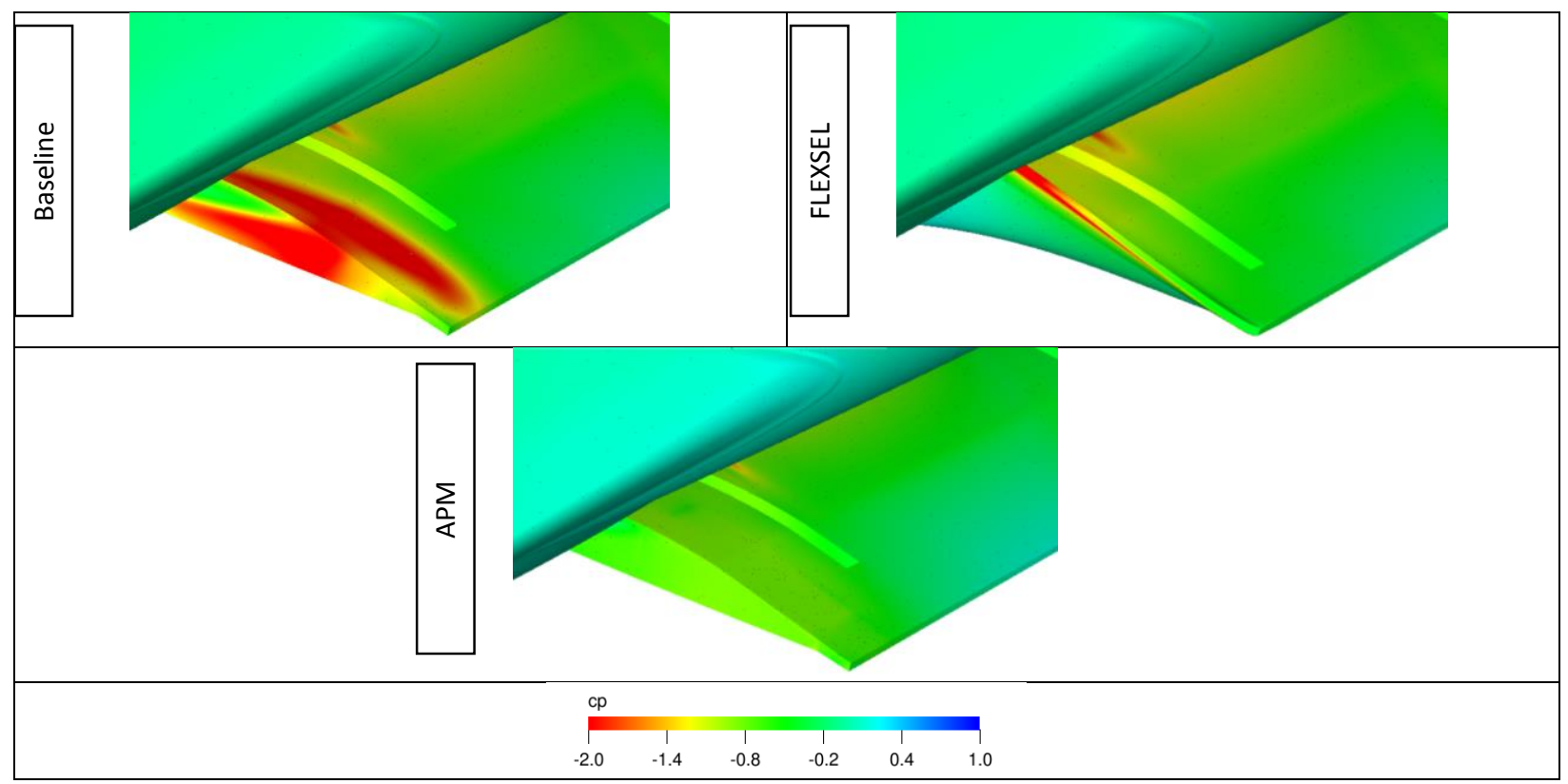

Figure 9. Comparison of static pressure distribution on the inboard flap side edge. 


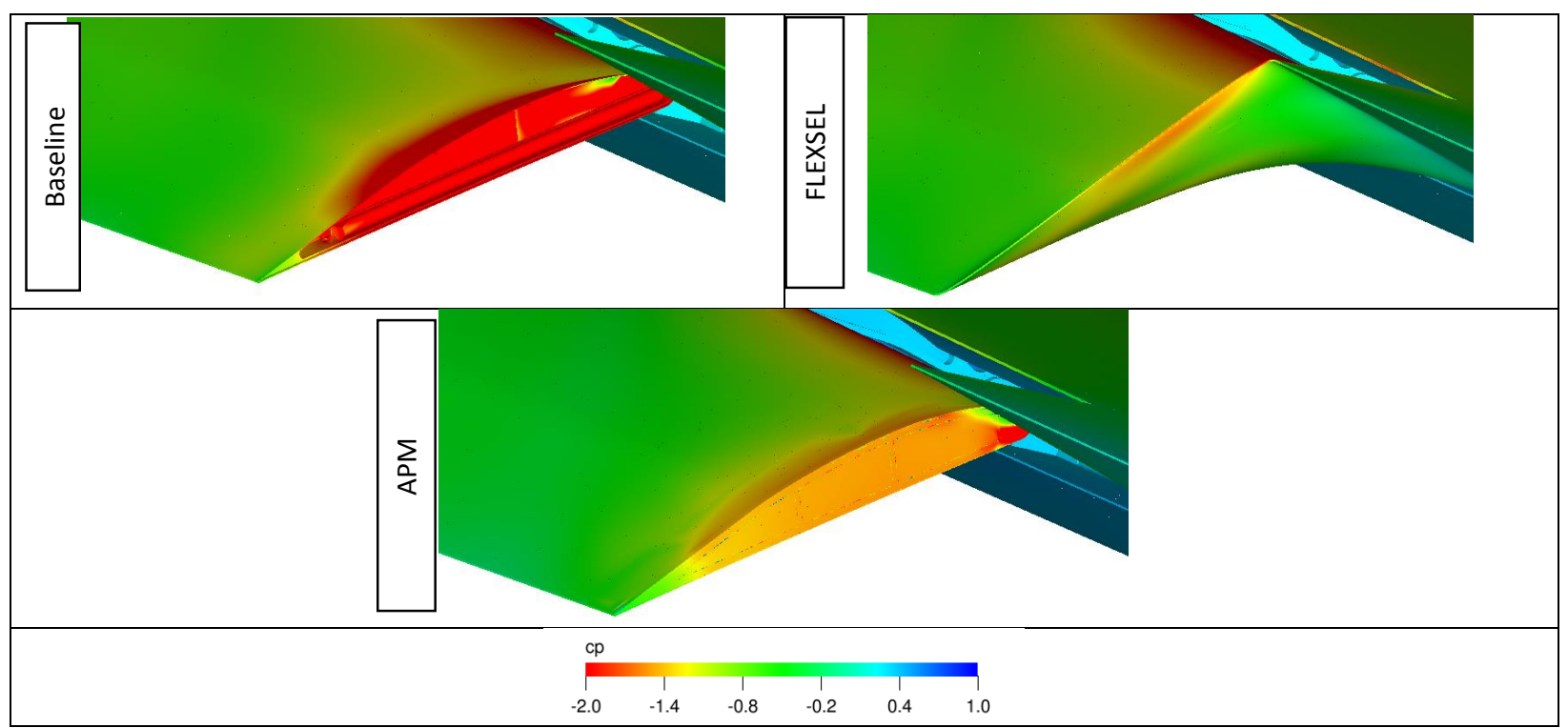

Figure 10. Comparison of static pressure distribution on the outboard flap side edge.

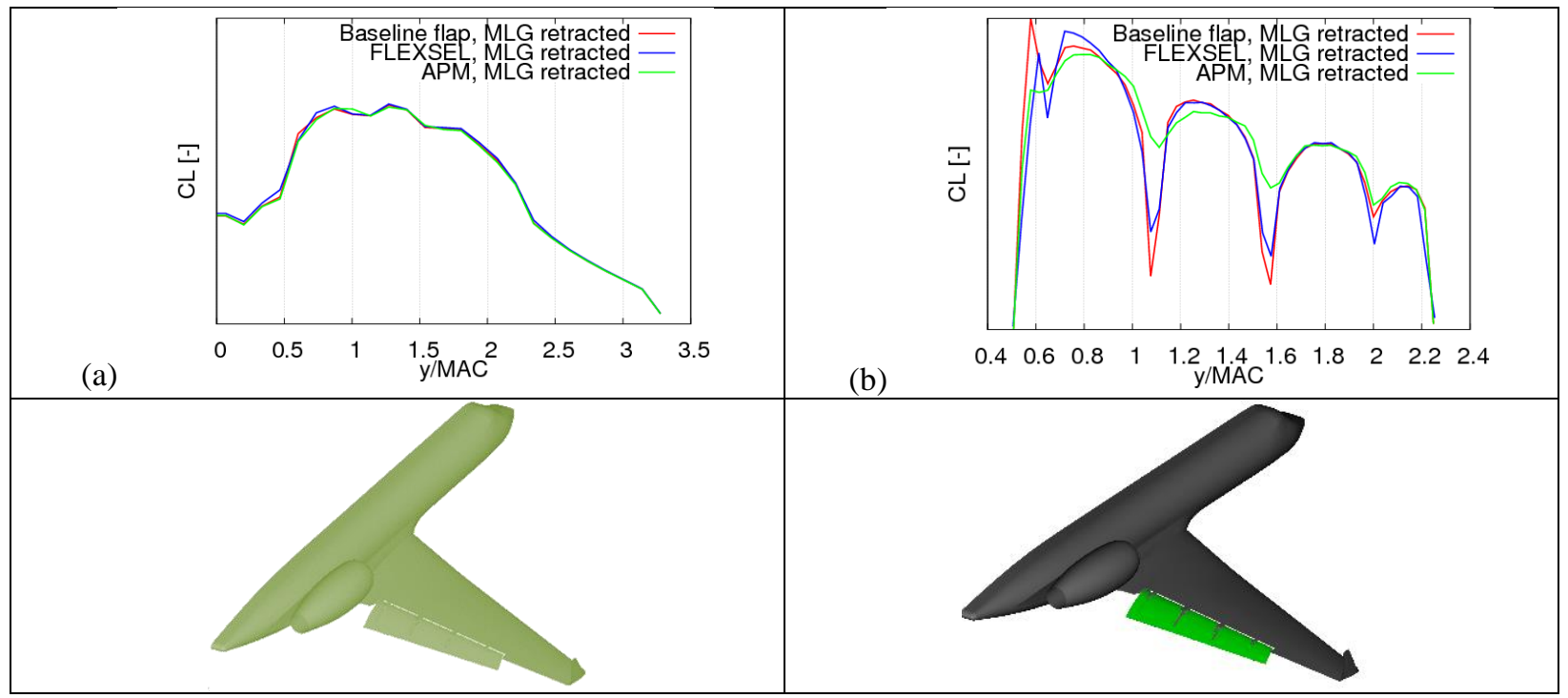

Figure 11. Comparison of the spanwise lift distribution on the half aircraft (left) and flap alone (right).
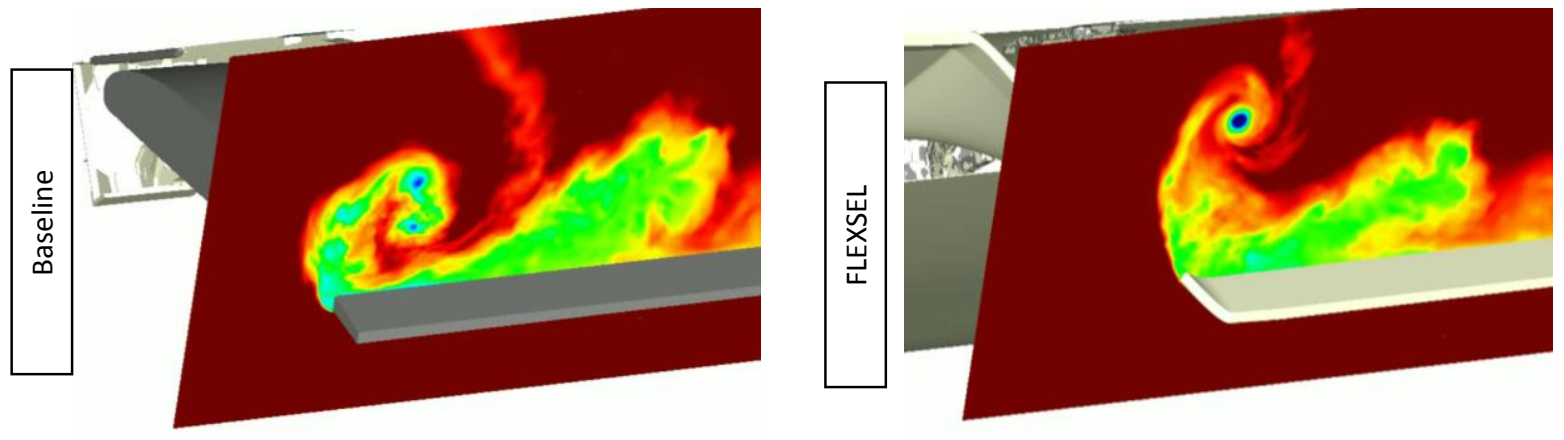

Figure 12. Flow structures snapshot at the inboard flap. 


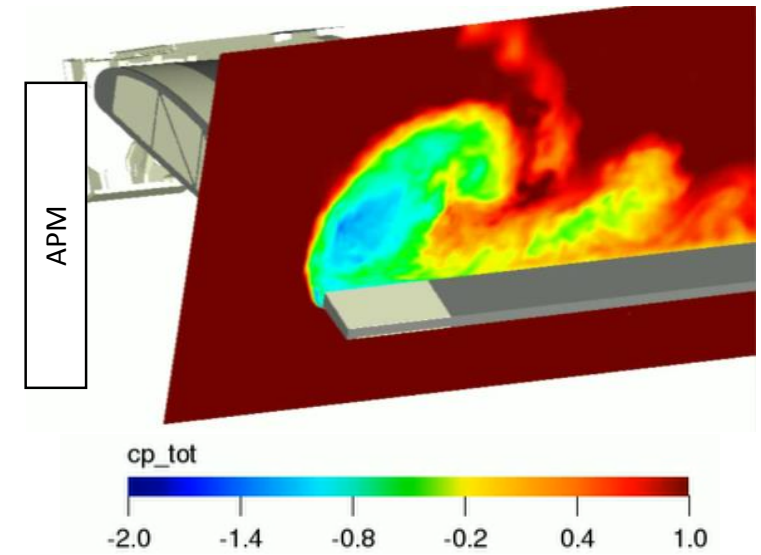

Figure 12. Concluded.

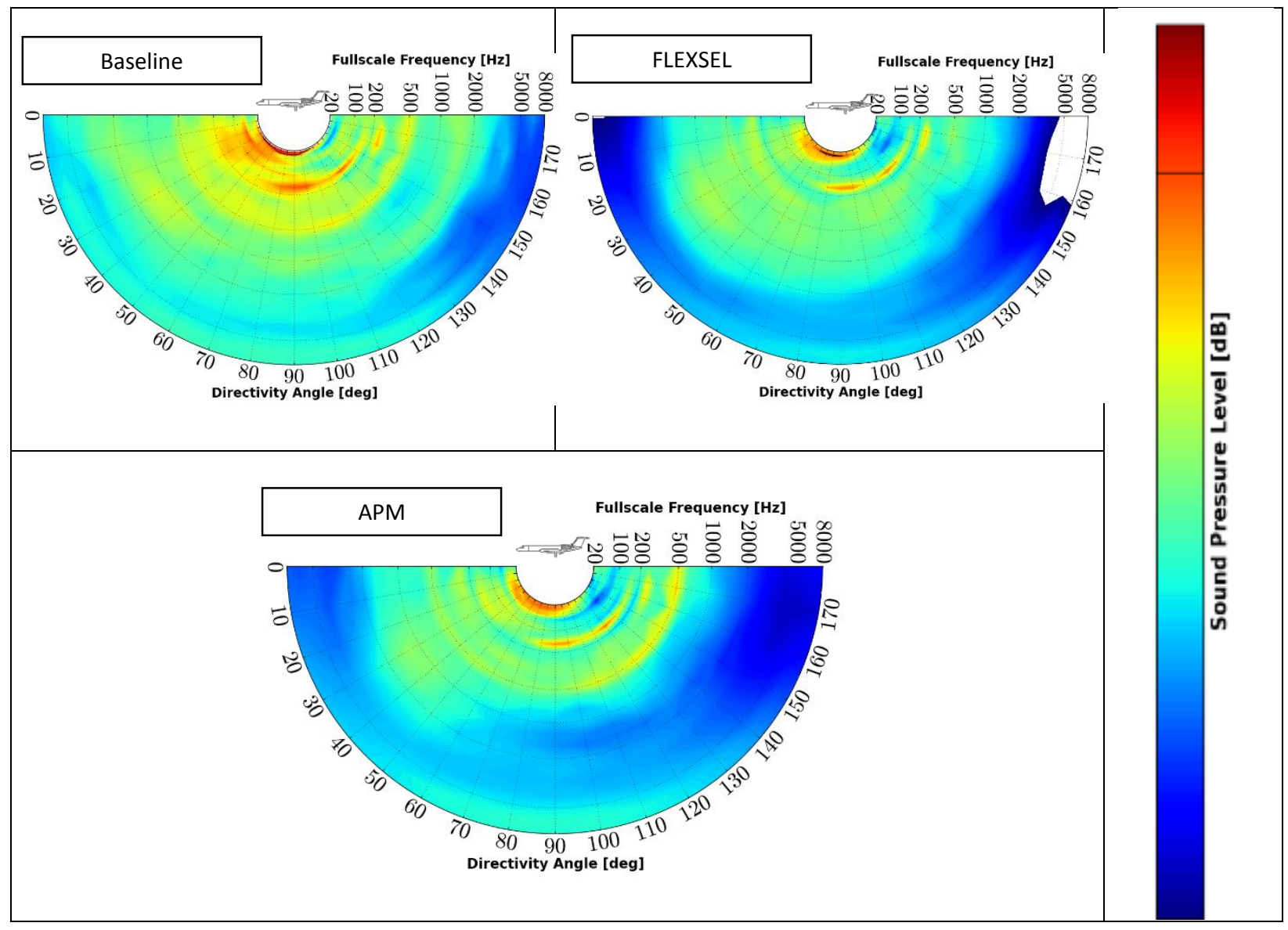

Figure 13. Farfield SPL directivity maps $\left(0^{\circ}\right.$ indicates aircraft forward flight direction). 


\section{Noise Reduction Concepts for Main Land- ing Gear}

We now focus on the configuration with retracted flap $\left(0^{\circ}\right.$ deflection $)$ and main landing gear (MLG) deployed. As mentioned in section II, this configuration has been used previously to study gear noise in isolation and to determine the acoustic performance of various noise mitigation technologies both in model-scale ground and full-sale flight tests. ${ }^{6,18,19}$ Figure 15 depicts the effect of the installed PKF and UF on the instantaneous velocity field surrounding the gear. The field was sampled on a vertical plane bisecting the front post and the rear shock strut. The porous knee fairing produces a much wider wake, thus immersing the front and rear posts in a slowly moving flow. Farfield SPL directivity maps are presented in Fig. 16. A low frequency peak near $40 \mathrm{~Hz}$, produced by the gear cavity, appears prominently in both maps. The fairings perform relatively well in alleviating forward-radiated noise. Predicted noise spectra for $90^{\circ}$ (overhead) at a distance of $394 \mathrm{ft}$., corresponding to the certification point, are plotted in Fig. 17. A prominent gear cavity tone at approximately $40 \mathrm{~Hz}$ is clearly visible. The spectrum of the treated gear shows that the fairings reduce gear noise for frequencies higher than $300 \mathrm{~Hz}$. The reduction in D-weighed OASPL produced by the fairings was close to $1.5 \mathrm{dBD}$.

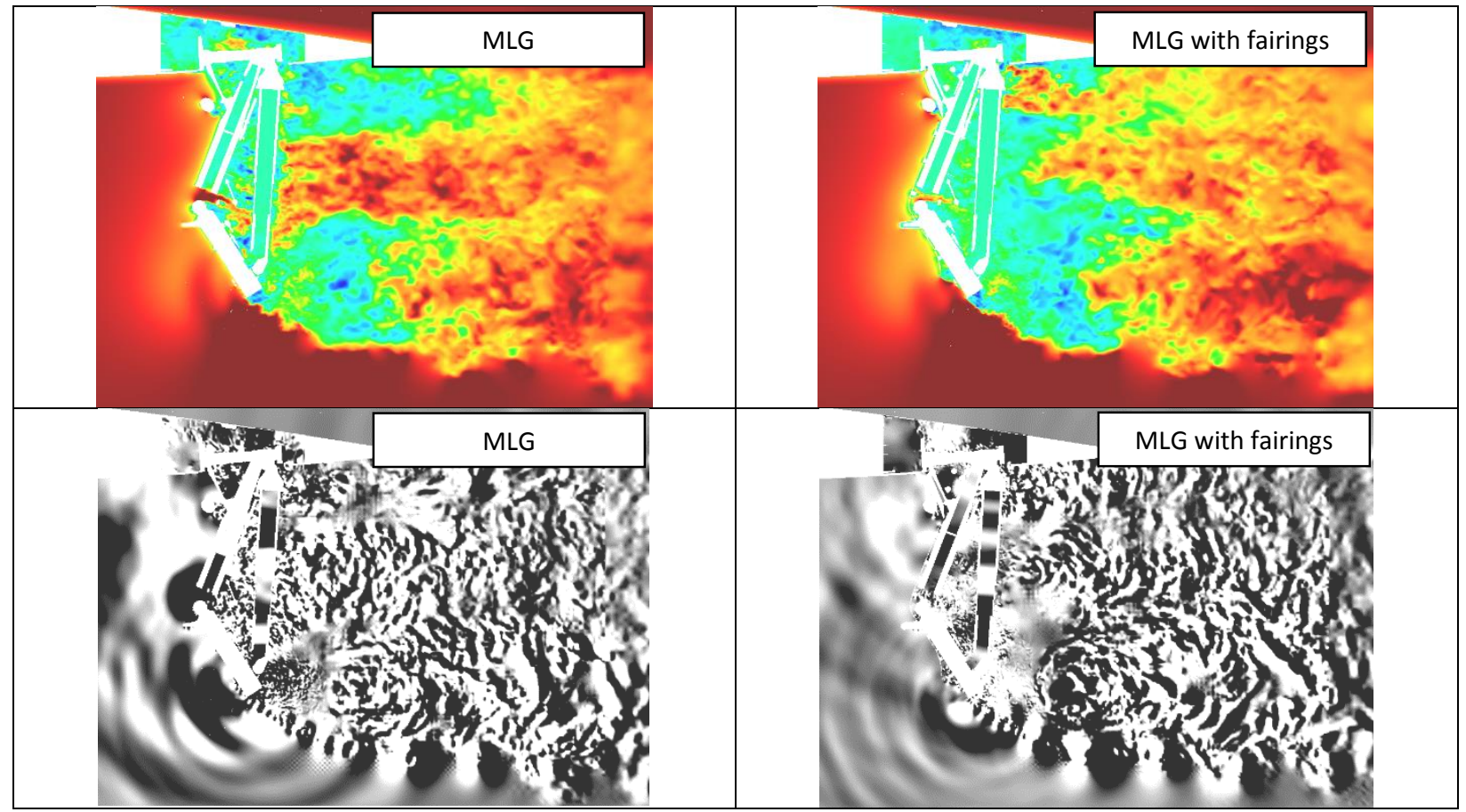

Figure 15. Instantaneous velocity field (top) and acoustic dilatation field (bottom) in a plane cutting vertically through the main landing gear.

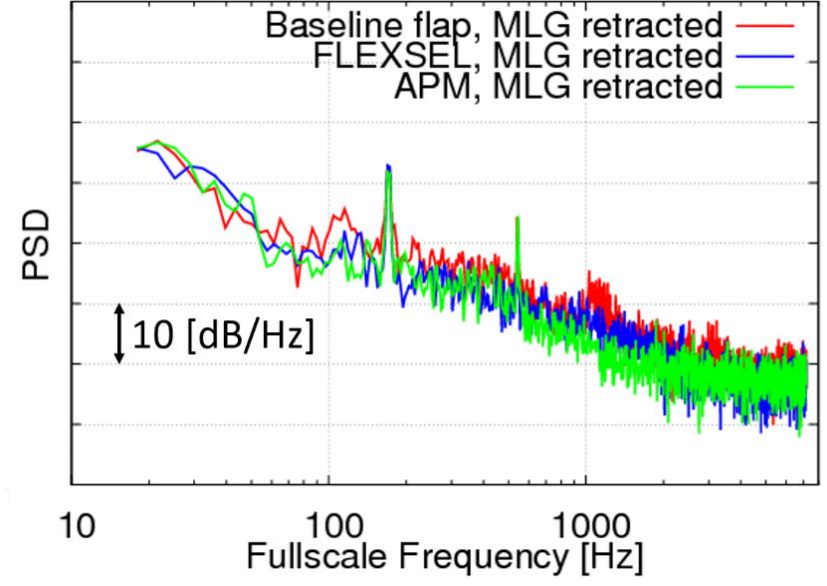

Figure 14. Farfield pressure spectra (overhead position). 

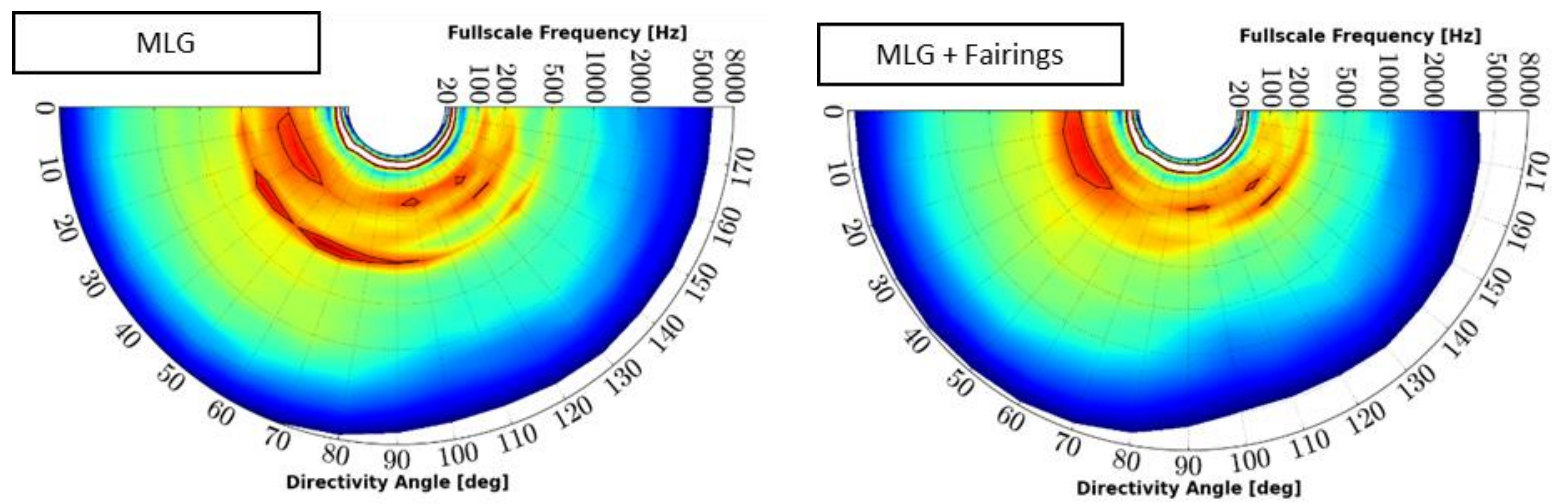

Sound Pressure Level [dB]

Figure 16. Farfield SPL directivity maps $\left(0^{\circ}\right.$ indicates aircraft forward flight direction).

\section{Combined Effect of Flap and Gear Noise Reduction Concepts}

Results for the landing configuration with flaps deflected $39^{\circ}$ and main gear deployed are presented in this section. Figure 18 shows the instantaneous, three-dimensional flow near the main landing gear and inboard flap side edge. Vortex cores are shown as $\lambda_{2}$ isosurfaces, which are colored by velocity magnitude and highlight the transient character of the flow. Aircraft solid surfaces are colored in gray scale by the pressure dilatation field. Close proximity of the flap and main gear causes strong interactions among the noise-generating mechanisms associated with these two airframe components. Given the complexities of the flow, only minor qualitative differences can be discerned among the three displayed snapshots. A better comparison is facilitated by the farfield di-

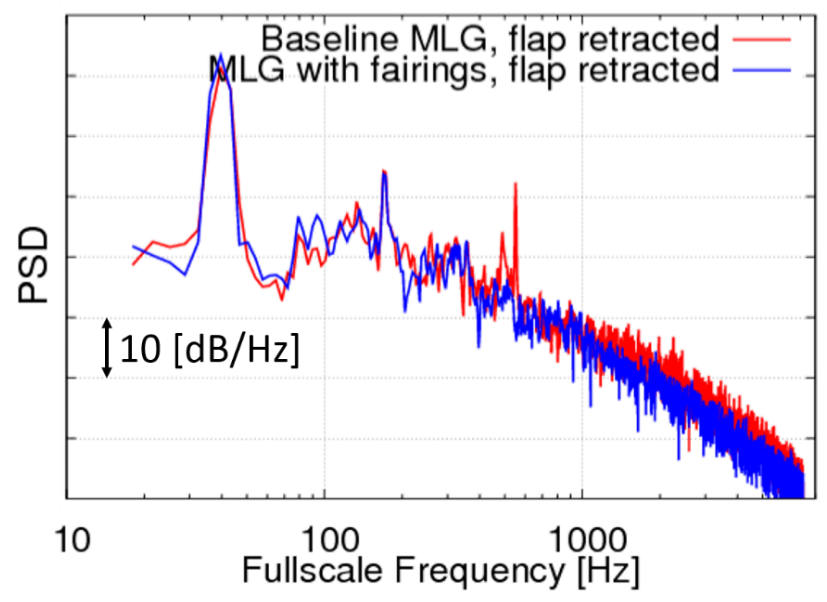

Figure 17. Farfield pressure spectra (overhead position). rectivity maps and overhead spectra shown in Figs. 19 and 20, respectively. All three maps in Fig. 19 depict strong forward noise radiation between $20^{\circ}$ and $60^{\circ}$. The baseline directivity map clearly shows the predominance of the tonal feature at $540 \mathrm{~Hz}$ that is associated with the small cavities on the port wing (Fig. 8). As expected, this tone was removed by the FLEXSEL concept. The same tone was also substantially diminished by the combination of gear fairings and porous surface treatment. The remaining differences in directivity for the three configurations considered here are subtle and best described via the flyover spectra of Fig. 20. Close scrutiny of the plots reveals that concurrent application of the flap and gear NR concepts heightens the tonal content of the spectra at frequencies between $100 \mathrm{~Hz}$ and $150 \mathrm{~Hz}$. These additional tones are presumably caused by alteration of the flow field at the landing gear cavity opening. A visual inspection of the spectra shown in Fig. 20 indicates that, relative to the individual components, lesser noise reduction benefits were achieved when the gear and flap are treated simultaneously. Based on D-weighted results, the reduction in OASPL produced by the combined effects of FLEXSEL and MLG fairings is close to $1.2 \mathrm{dBD}$; for the concurrent application of the porous surface (APM) treatment and MLG fairings, the reduction is approximately $1.6 \mathrm{dBD}$. These reduction levels clearly suggest that gear-flap interaction (installation) effects may strongly influence the aeroacoustic performance of noise abatement technologies. Also important is the presence of gear cavity noise at low frequencies and flap bracket noise at high frequencies that mask the true performance of the technologies being evaluated. 


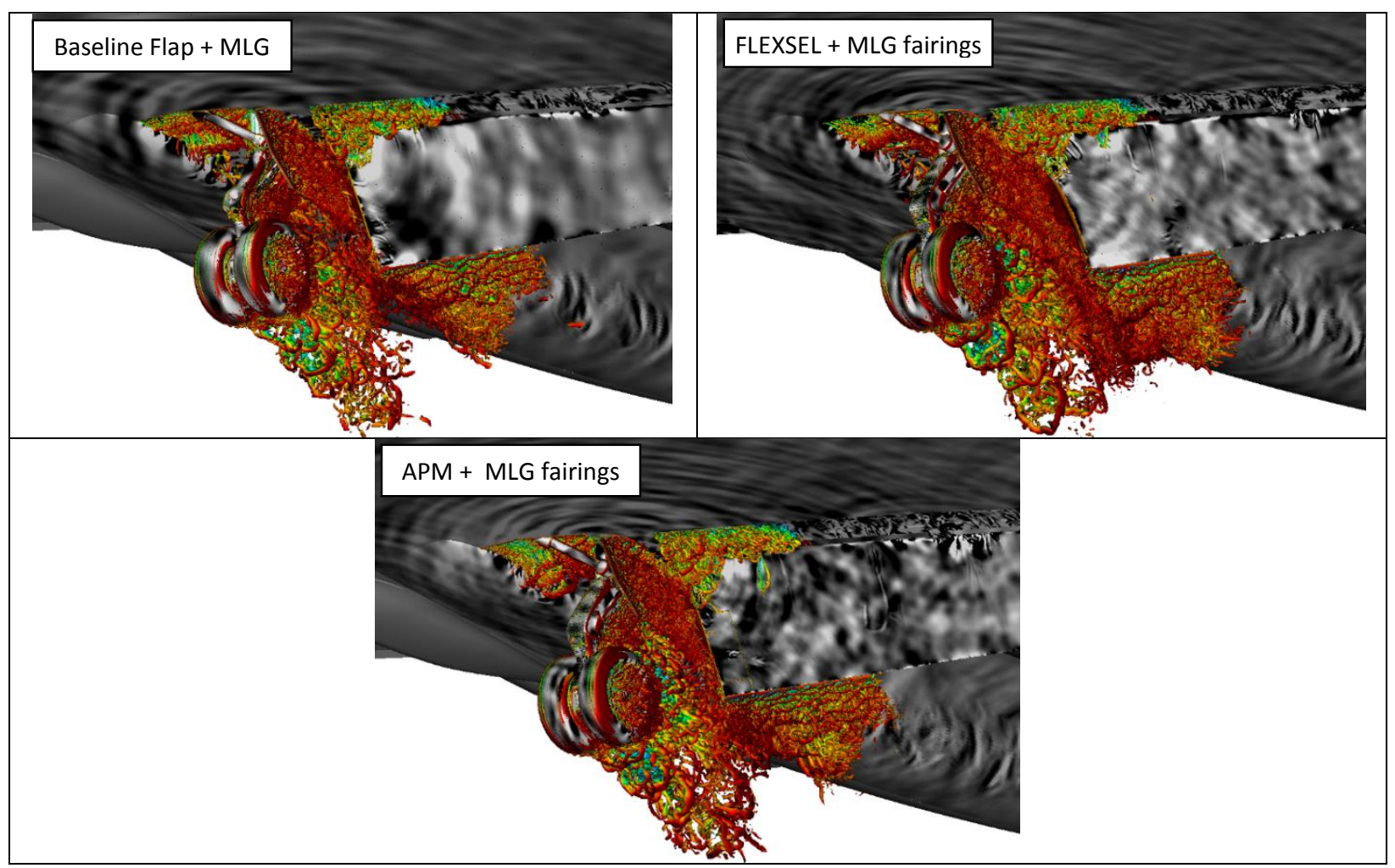

Figure 18. Snapshot of the three-dimensional flow near the main landing gear and inboard flap side edge.

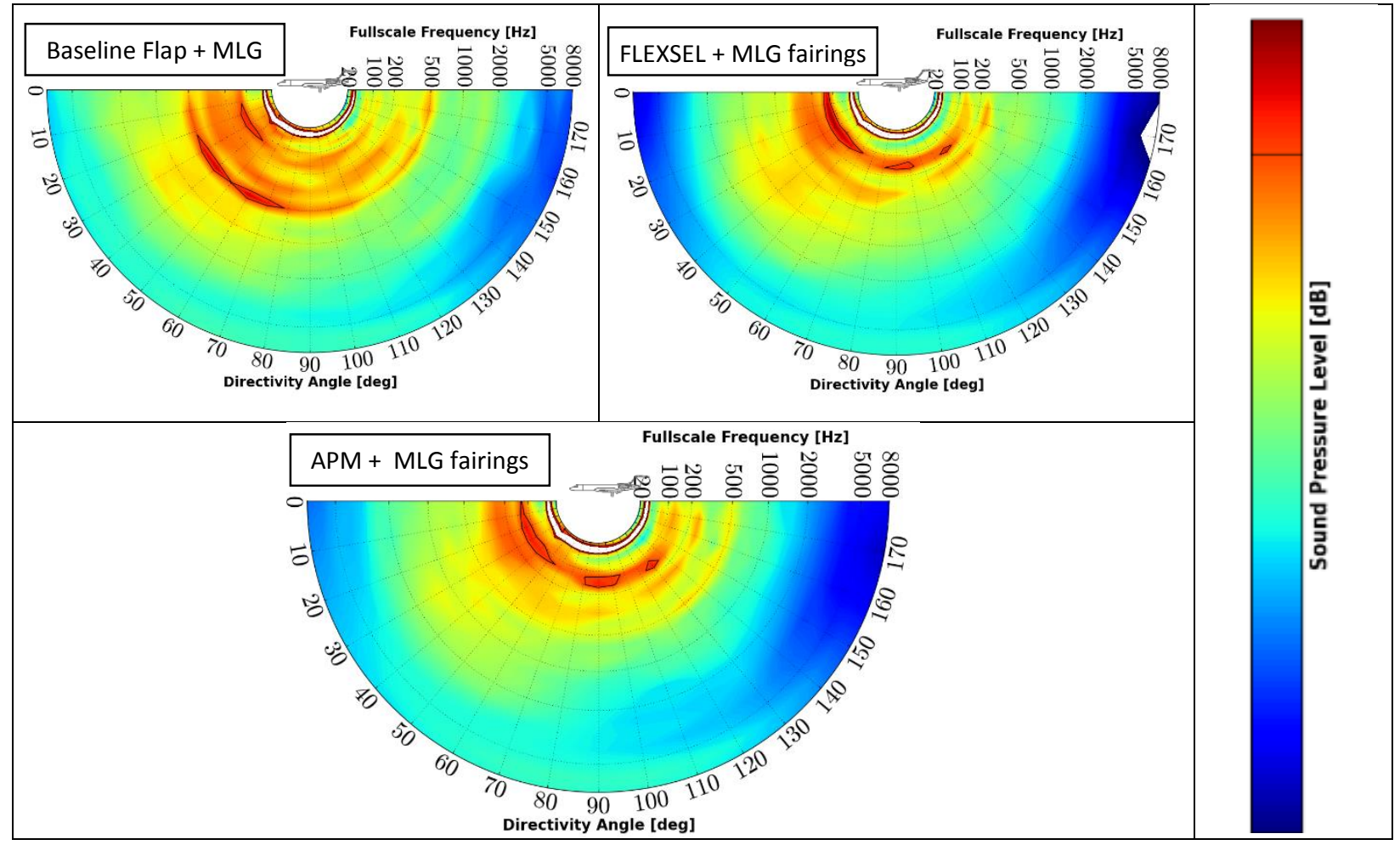

Figure 19. Farfield SPL directivity maps $\left(0^{\circ}\right.$ indicate aircraft forward flight direction). 
Since the reduction of community noise is the ultimate goal of this investigation, perceived noise levels (PNL) for an aircraft flying over an observer on the ground are shown in Fig. 21. The standard Federal Aviation Administration (FAA) landing trajectory for certification was used for these calculations. Compared to the baseline configuration, a moderate level of noise reduction is observed for both NR concepts in combination with the MLG fairings. In terms of effective perceived noise level (EPNL), a reduction of 1.2 EPNdB was achieved for combined FLEXSEL - MLG fairings and 1.0 EPNdB for combined APM - MLG fairings. Ground maps of maximum PNL are shown in Fig. 22 for the three configurations during an approach to London Heathrow airport. The impact of the noise reduction concepts on the aircraft acoustic footprint (defined by iso-lines of constant PNL) is clearly seen in the maps.

\section{Concluding Remarks}

The present work documents a detailed computational study aimed at evaluating the systemlevel aeroacoustic performance of a select number of flap and main gear NR technologies applied to a full-scale, complete aircraft during landing. To accomplish this ambitious goal, the computational effort was centered on conducting high-fidelity, time-accurate simulations of a full-scale Gulfstream aircraft that features nearly all of the finer details for the flap and main landing gear components.

The Exa Corporation PowerFLOW ${ }^{\circledR}$ lattice Boltzmann solver was used to perform the computations for free air at a Mach number of 0.2 and a $\mathrm{Re}$ of $10.5 \times 10^{6}$ representing half of the flight $\mathrm{Re}$ during aircraft landing. Validated simulation results for three baseline (untreated) configurations were used to determine the effectiveness of various flap and main landing gear NR concepts as applied to the aircraft individually and concurrently. The flap concepts evaluated in the present study comprised a porous surface treatment applied to small segments at the inboard and outboard flap tips and FLEXSEL, a technology that structurally links a flap side edge to the adjacent wing edge. For the main landing gear, the concepts studied comprised a porous knee fairing (PKF) and an assortment of smaller fairings that are collectively termed as "upper fairings" (UF). Except for FLEXSEL, the devices were within those downselected for implementation on the Gulfstream test aircraft after the Gulfstream-NASA critical design review process.

The configuration with flaps deflected $39^{\circ}$ and main gear retracted was used to determine the aeroacoustic performance of the porous surface treatment and FLEXSEL concepts on a component-level basis. Predicted SPL directivity maps and farfield noise spectra at the overhead position (corresponding to a standard aircraft certification point on approach) were examined to assess the effectiveness of each flap technology. Applying D-weighting to the spectra, a noise reduction benefit of approximately $2.5 \mathrm{dBD}$ in OASPL relative to the untreated (baseline) flap configuration was obtained for each concept. The noise mitigation capability of the main gear concepts was predicted using the configuration with retracted $\left(0^{\circ}\right)$ flap and main gear deployed. The fairings were found to diminish radiated gear noise over a broad frequency range, resulting in a $1.5 \mathrm{dBD}$ reduction in OASPL relative to the baseline configuration. The configuration with flaps deflected $39^{\circ}$ and main gear deployed, representing a landing aircraft, was used to evaluate the acoustic performance of flap and gear concepts when applied simultaneously. For the combination of treatments involving FLEXSEL plus gear fairings, D-weighting of the farfield spectra at the overhead certification point yielded a $1.2 \mathrm{dBD}$ reduction in OASPL. The reduction in OASPL for the combination of porous tip surface and gear fairings 
was determined to be $1.6 \mathrm{dBD}$. However, the noise reduction potential of the present flap and gear technologies is expected to be substantially higher. Targeted studies presented in companion papers have revealed that the true acoustic performance of the current technologies is masked by the presence of excess low-frequency noise produced by the gear cavity and excess high-frequency noise produced by the flap brackets.

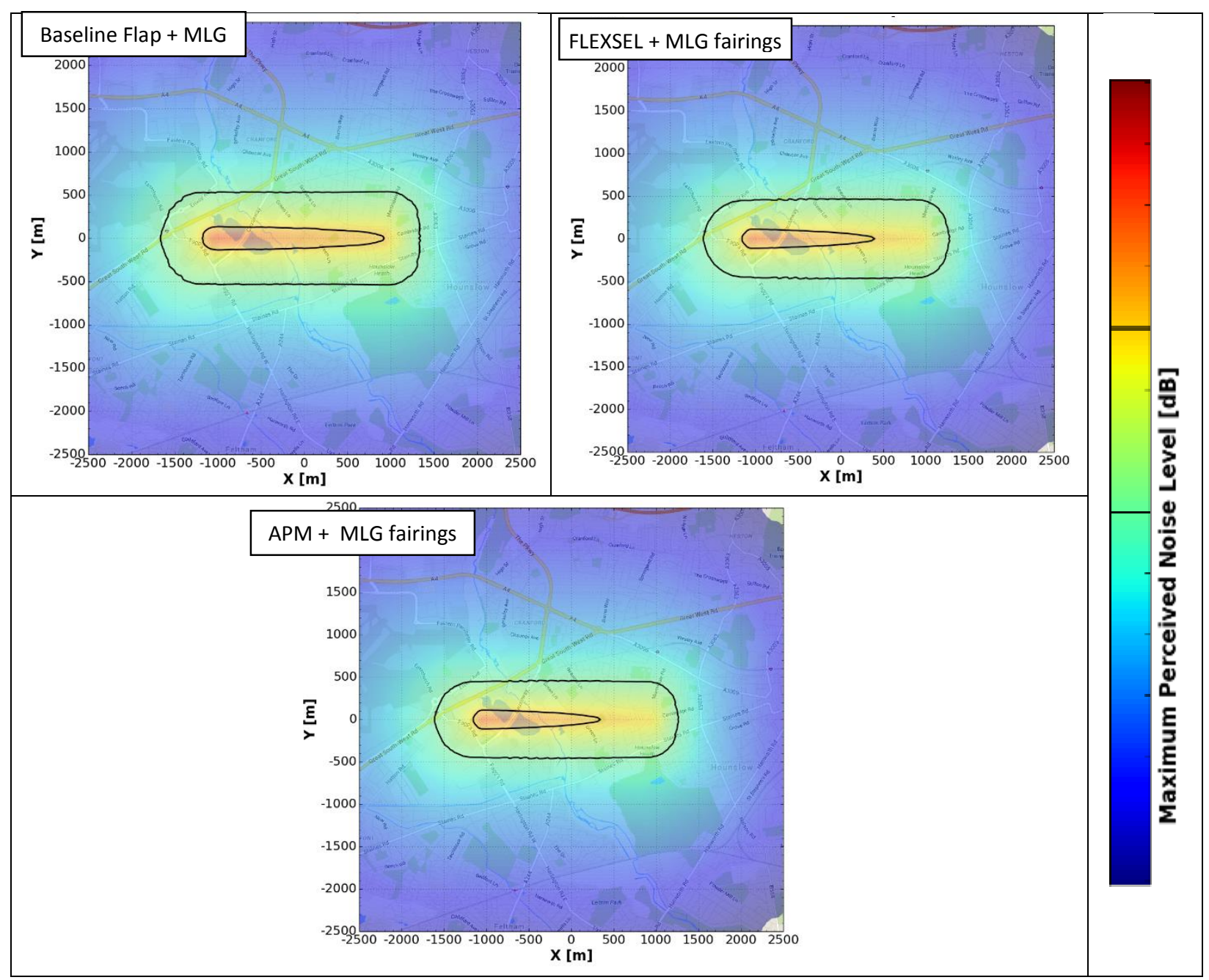

Figure 22. Maps of maximum PNL on the ground.

\section{Acknowledgments}

This work was supported by the Environmentally Responsible Aviation (ERA) project under the Integrated Aviation Systems Program (IASP) of NASA. Special thanks are due to Thomas Van de Ven (retired) and Scott Dutton of GAC for facilitating, and assisting with, the transfer and development of the full-scale aircraft geometry model. Our thanks also go to Scott Brynildsen of Vigyan, Inc. for providing geometry modifications and CAD support. We would also like to express our sincere appreciation to Patrick Moran of NASA Ames Research Center for high-quality visualizations and animations of the large data sets. All the simulations were performed on the Pleiades supercomputer at the NASA Advanced Supercomputing (NAS) facility at Ames Research Center. The logistical support provided by NAS staff, in particular Yan-Tyng (Sherry) Chang of Computer Sciences Corporation, is greatly appreciated.

\section{References}

${ }^{1}$ Dobrzynski, W., "Almost 40 Years of Airframe Noise Research: What Did We Achieve," J. Aircraft, Vol. 47, No. 2, March-April 2010, pp. 353 - 367. 
${ }^{2}$ Michel, U., Barsikow, B., Helbig, J., Hellmig, M., and Schüttpelz, M., "Flyover Noise Measurements on Landing Aircraft with a Microphone Array," AIAA Paper 98-2336, May 1998.

${ }^{3}$ Piet, J. F., Elias, G., and Lebigot, P., "Localization of Acoustic Sources from a Landing Aircraft with a Microphone Array," AIAA Paper 99-1811, May 1999.

${ }^{4}$ Stoker, R., Guo, Y., Streett, C. L., and Burnside, N., "Airframe Noise Source Locations of a 777 Aircraft in Flight and Comparisons with Past Model Tests," AIAA Paper 2003-3232, May 2003.

${ }^{5}$ Brusniak, L., Underbrink, J. R., and Stoker, R. W., "Acoustic Imaging of Aircraft Noise Sources Using Large Aperture Phased Arrays," AIAA Paper 2006-2715, May 2006.

${ }^{6}$ Elkoby, R., Brusniak, L., Stoker, R., Khorrami, M. R., Abeysinghe, A., and Moe, J. W., "Airframe Noise Results from the QTD II Flight Test Program,” AIAA Paper 2007-3457, May 2007.

${ }^{7}$ Li. Y., Satti, R., Lew, P.-T., Shock, R., and Noelting, S., "Computational Aeroacoustic Analysis of Flow Around a Complex Nose Landing Gear Configuration," AIAA Paper 2008-2916, May 2008.

${ }^{8}$ Keating, A., Dethioux, P., Satti, R., Noelting, S., Louis, J., Van de Ven, T., and Vieito, R., "Computational Aeroacoustics Validation and Analysis of a Nose Landing Gear," AIAA Paper 2009-3154, May 2009.

${ }^{9}$ Fares, E. and Noelting, S., "Unsteady Flow Simulation of a High-Lift Configuration using a Lattice-Boltzmann Approach,” AIAA Paper 2011-0869, January 2011.

${ }^{10}$ Vatsa, V., Lockard, D. P., Khorrami, M. R., and Carlson, J.-R., "Aeroacoustic Simulation of a Nose Landing Gear in an Open-Jet Facility using FUN3D," AIAA Paper 2012-2280, June 2012.

${ }^{11}$ Casalino, D., Noelting, S., Fares, E., Vand de Ven, T., Perot, F., and Bres, G., "Towards Numerical Aircraft Noise Certification: Analysis of a Full-Scale Landing Gear in Fly-Over Configuration," AIAA Paper 2012-2235, June 2012.

${ }^{12}$ Khorrami, M. R. and Mineck, R. E., "Towards Full Aircraft Airframe Noise Prediction: Detached Eddy Simulations," AIAA Paper 2014-2480, June 2014.

${ }^{13}$ Khorrami, M. R., Fares, E., and Casalino, D., "Towards Full-Aircraft Airframe Noise Prediction: Lattice-Boltzmann Simulations," AIAA Paper 2014-2481, June 2014.

${ }^{14}$ Fares, E., and Casalino, D., and Khorrami, M. R., "Evaluation of Airframe Noise Reduction Concepts via Simulations using a Lattice-Boltzmann Approach," AIAA Paper 2015-2988, June 2015.

${ }^{15}$ Khorrami, M. R., Mineck, R. E., Yao, C. S., and Jenkins, L. N., "A Comparative Study of Simulated and measured Gear-Flap Flow Interaction,” AIAA Paper 2015-2989, June 2015.

${ }^{16}$ Fares, E., Duda, B., and Khorrami, M. R., "Airframe Noise Prediction of a Full Aircraft in Model and Full Scale Using a Lattice Boltzmann Approach," Paper to be presented at the $22^{\text {nd }}$ AIAA/CEAS Aeroacoustics Conference in Lyon, France,, May-June 2016.

${ }^{17}$ Khorrami, M. R. and Fares, E., "Simulation - Based Airframe Noise Prediction of a Full - Scale, Full Aircraft," Paper to be presented at the 22 ${ }^{\text {nd }}$ AIAA/CEAS Aeroacoustics Conference in Lyon, France, May-June 2016.

${ }^{18}$ Khorrami, M. R., Lockard, D. P., Humphreys, Jr., W. M., Choudhari, M. M., and Van de Ven, T., "Preliminary Analysis of Acoustic Measurements from the NASA-Gulfstream Airframe Noise Flight Test," AIAA Paper 20082814, May 2008.

${ }^{19}$ Khorrami, M. R., Humphreys, W. M. Jr., Lockard, D. P., and Ravetta, P. A., “Aeroacoustic Evaluaion of Flap and Landing Gear Reduction Concepts," AIAA Paper 2014-2478, 2014.

${ }^{20}$ Chen, H., Chen, S., and Matthaeus, W., "Recovery of the Navier-Stokes Equations Using a Lattice-Gas Boltzmann Method," Physical Review A, Vol. 45, No. 8, 1992, pp.5339- 5342.

${ }^{21}$ Lockard, D. "Summary of the Tandem Cylinder Solutions from the Benchmark problems for Airframe Noise Computations-I Workshop," AIAA Paper 2011-0353, 2011.

${ }^{22}$ Yakhot, V. and Orszag, S. A., "Renormalization Group Analysis of Turbulence. I. Basic Theory," J. Sci. Comput., Vol 1, No. 2, 1986, pp. $3-51$.

${ }^{23}$ Chen, H., Kandasamy, S., Orszag, S.A., Succi, S., and Yakhot, V., "Extended Bolzmann Kinetic Equation for Turbulent Flows," Science, Vol. 301, No. 5633, 2003, pp. 633 - 636.

${ }^{24}$ Chen J., "Volumetric formulation of the lattice Boltzmann method for fluid dynamics: Basic concept," Physical Review E, vol. 58, no.3, 1998, pp. $3955-3963$. 
${ }^{25}$ Chen, H., Teixeira, C., and Molvig, K., "Realization of Fluid Boundary Conditions via Discrete Boltzmann Dynamics," International Journal of Modern Physics C, Vol. 9, No. 8, 1998, pp. 1281 - 1292.

${ }^{26}$ Pérot, F., Freed, D., and Mann, A., “Acoustic Absorption of porous materials using LBM”, AIAA Paper 20132070, 2013.

${ }^{27}$ Mann, A., Pérot, F., Kim, M., and Casalino, D., "Characterization of Acoustic Liners Absorption using a LatticeBoltzmann Method”, AIAA Paper 2013-2271, 2013.

${ }^{28}$ Freed, D. M., "Lattice-Boltzmann Method for Macroscopic Porous Media Modeling," International Journal of Modern Physics, Vol. 9, 1998, pp. 1491 -1505.

${ }^{29}$ Sun, C., Pérot, F. L., Zhang, R., Chen, H., Freed, D. M., and Staroselsky, I., "Computer Simulation of Physical Processes," US Patent App. 13/292,844, May 9, 2013.

${ }^{30}$ Pérot, F. L., Freed, D. M., Mann A., “Acoustic Absorption of Porous Materials Using LBM”, AIAA paper 20132070, 2013.

${ }^{31}$ Mann, A., Pérot, F., Kim, M., and Casalino, D., "Characterization of Acoustic Liners Absorption using a LatticeBoltzmann Method”, AIAA Paper 2013-2271, 2013.

${ }^{32}$ Ffowcs Williams, J. E. and Hawkings, D. L., "Sound Generated by Turbulence and Surfaces in Arbitrary Motion," Philosophical Transactions of the Royal Society, Vol. A264, No. 1151, 1969, pp. 321 - 342.

${ }^{33}$ Farassat, F. and Succi, G. P., "The Prediction of Helicopter Discrete Frequency Noise," Vertica, Vol. 7, No. 4, 1983, pp. $309-320$.

${ }^{34}$ Najafi-Yazdi, A., Brès, G. A., and Mongeau, L., "An Acoustic Analogy Formulation for Moving Sources in Uniformly Moving Media," Proceedings of The Royal Society of London A, Vol. 467 (2125), 2011, pp. 144 - 165.

${ }^{35}$ Neuhart, D. H., Hannon, J. A., and Khorrami, M. R., "Aerodynamic Measurements of a Gulfstream Aircraft Model with and without Noise Reduction Concepts," AIAA Paper 2014-2477, June 2014. 